\title{
Erratum to: Pandemic and seasonal vaccine coverage and effectiveness during the 2009-2010 pandemic influenza in an Italian adult population
}

Simona Costanzo - Francesco Gianfagna - Mariarosaria Persichillo Francesca De Lucia $\cdot$ Angelita Verna $\cdot$ Modjenar Djidingar $\cdot$ Sara Magnacca $\cdot$ Francesca Bracone $\cdot$

Marco Olivieri · Maria Benedetta Donati · Giovanni de Gaetano $\cdot$ Licia Iacoviello

Published online: 3 May 2012

(C) Swiss School of Public Health 2012

Erratum to: Int J Public Health

DOI 10.1007/s00038-012-0346-0

Unfortunately, an author's name was rendered wrongly.

Instead of Francesca D Lucia it should read: Francesca

De Lucia.

The online version of the original article can be found under doi:10.1007/s00038-012-0346-0.

S. Costanzo - F. Gianfagna - M. Persichillo - F. De Lucia

A. Verna - M. Djidingar - S. Magnacca $\cdot$ F. Bracone

M. Olivieri · M. B. Donati · G. de Gaetano - L. Iacoviello ( $\square)$

Laboratory of Genetic and Environmental Epidemiology,

Research Laboratories, Fondazione di Ricerca

e Cura 'Giovanni, Paolo II', Largo Gemelli 1, 86100

Campobasso, Italy

e-mail: licia.iacoviello@moli-sani.org 